\title{
CHARMONIUM PRODUCTION AT HERA
}

\author{
ANDREAS B. MEYER \\ DESY, Notkestr.85, 22603 Hamburg, Germany \\ andreas.meyer@desy.de
}

\begin{abstract}
Measurements of Charmonium production at the electron proton collider HERA are presented. In inelastic production the charmonium is formed from $c \bar{c}$ pairs that are produced in photon gluon fusion. The measurements of the cross sections and helicity distributions are compared to calculations performed in the framework of non-relativistic quantum chromodynamics (NRQCD), which includes both color-singlet and color-octet contributions.
\end{abstract}

Keywords: Charmonium; HERA

PACS numbers: 11.25.Hf, $123.1 \mathrm{~K}$

\section{Introduction}

The electron proton storage ring HERA, at DESY Hamburg, collides electrons (or positrons) with an energy of $27.5 \mathrm{GeV}$ with protons of $920 \mathrm{GeV}$, yielding a ep centerof-mass energy of $\sim 320 \mathrm{GeV}$. HERA has been in operation since 1992. During the running period HERA-I (1992-2000) the two experiments H1 and ZEUS collected an integrated luminosity of $100 \mathrm{pb}^{-1}$ each. After a luminosity upgrade of HERA (2001/02) the present data taking period HERA-II is scheduled until mid 2007, when an integrated luminosity, useful for physics analyses, of $\sim 500 \mathrm{pb}^{-1}$ is expected to be reached for each of the two experiments.

The H1 and ZEUS detectors are multi-purpose cylindrical devices, comprising tracking chambers and calorimeters, concentrically arranged around the HERA beams. A sketch of the $\mathrm{H} 1$ experiment (sideview) is given in fig. 2. Both experiments have published measurements of $J / \psi$ production cross sections in photoproduction and electroproduction based on HERA-I data which are presented in the following. The cross section results are complemented by measurements of $J / \psi$ decay angular distributions which give access to the $J / \psi$ polarization states.

\section{Experimental Environment}

Heavy quark production in ep collisions occurs dominantly through photon-gluon fusion where a photon from the incoming electron and a gluon from the proton produce a $c \bar{c}$ pair which hadronizes into a final state containing heavy hadrons, dominantly $D$-Mesons. More rarely, the $c \bar{c}$ pair recombines to form a charmonium 
2 Andreas B.Meyer

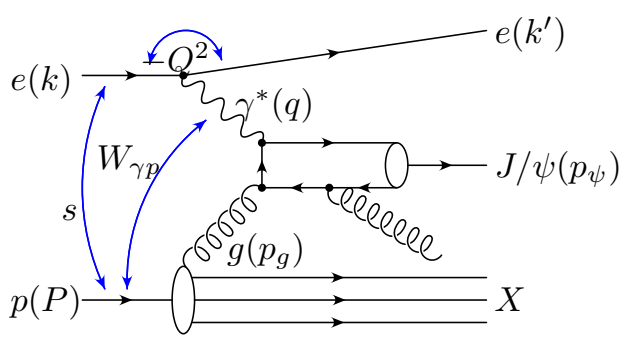

Fig. 1. Charmonium production in ep collisions via the photon-gluon-fusion process.

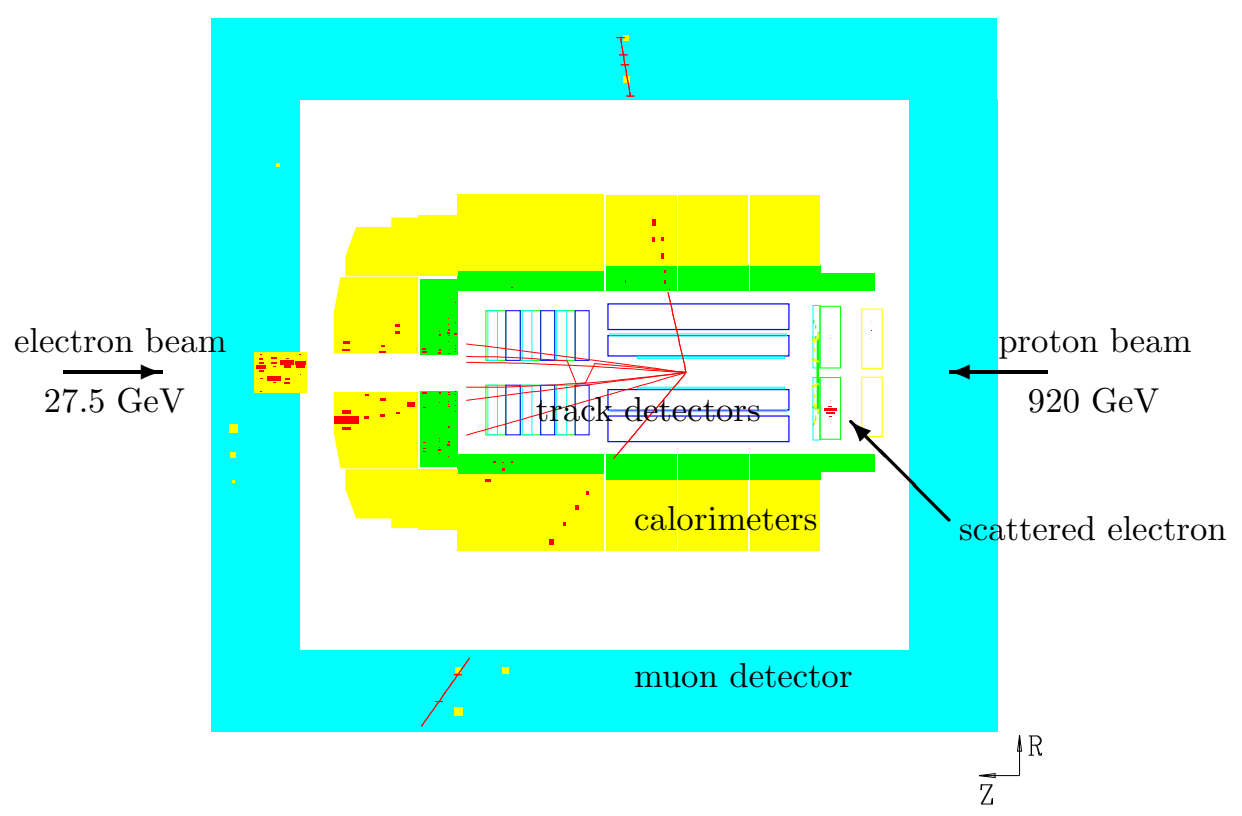

Fig. 2. Sketch of a $J / \psi$ candidate event in the H1 Experiment.
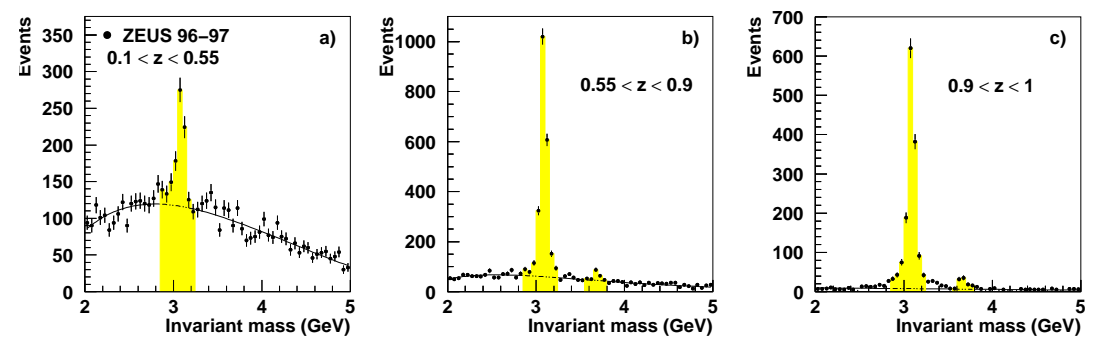

Fig. 3. $J / \psi$ signal distributions in three regions of elasticity $z$ as seen in the ZEUS data collected in the years 1994-1997. 


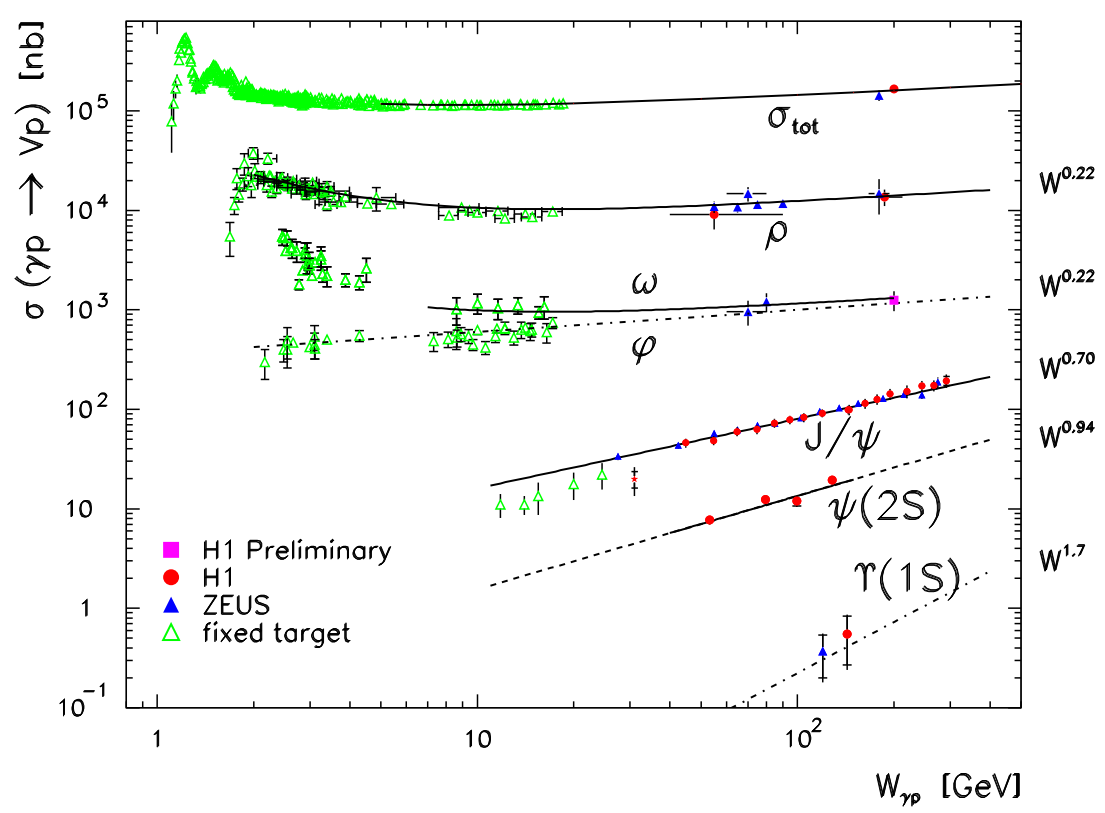

Fig. 4. Total cross section and cross sections for production of various vector mesons in $\gamma p$ collisions as a function of $W_{\gamma p}$, as measured at HERA and in fixed-target experiments.

state (fig. 1). In photoproduction, the photon virtuality $Q^{2}$ is small $\left(Q^{2} \sim 0\right)$ and the photon is quasi-real. In this regime, in addition to direct photon-gluon fusion, also photon-resolved processes contribute, in which the photon fluctuates into a hadronic state which subsequently interacts with the proton. In electroproduction, or deep inelastic $e p$-scattering (DIS), the exchanged photon has a non-zero virtuality $Q^{2} \gtrsim 1 \mathrm{GeV}^{2}$ and the electron scattering angle is large enough for the electron to be detected in the main detectors.

A sketch of a candidate event of inelastic $J / \psi$ production and its decay into two muons in the H1 Experiment is shown in fig. 2. The scattered electron candidate is visible in the backward calorimeter (electron beam direction). Additional final state particles are produced mostly in the forward direction (proton beam direction).

The fraction of the photon energy that is transferred from the incoming photon to the $J / \psi$ meson is estimated by the elasticity variable $z$. It is defined as $z=$ $\frac{\left(E-p_{z}\right)_{J / \psi}}{\left(E-p_{z}\right)_{\text {hadrons }}}$ where $E$ and $p_{z}$ in the numerator are the energy and longitudinal momentum component in the proton beam direction of the $J / \psi$, and $E$ and $p_{z}$ in the denominator are summed over the energies and $p$-beam longitudinal momentum components of all final state particles (except the scattered electron).

Figure 3 shows the signal distributions as obtained in the analysis of the data collected between 94 and 97 by the ZEUS collaboration ${ }^{1}$. Towards low values of $z$ the combinatorial background increases as more particles are produced in the final state. At $z \sim 1$ the dominant production process is diffractive elastic $J / \psi$ 
production in which a colorless state (to lowest order QCD consisting of two gluons) is exchanged between the proton and the $c \bar{c}$ pair. At HERA, this channel is dominant for quarkonia with quantum numbers of real photons (i.e. $J^{P C}=1^{--}$), such as the $J / \psi$ and $\psi(2 S)$. An overview of the energy dependence of the elastic quarkonium photoproduction cross section is shown in fig. 4 . For further details on elastic vector meson production we refer to a presentation at Meson $2004^{2}$.

\section{Theory of Charmonium Production}

Many models have been suggested to describe inelastic charmonium production in the framework of perturbative QCD, such as the color-singlet model (CSM) ${ }^{3}$, the color-evaporation model ${ }^{4}$ and soft color interactions ${ }^{5}$. Most recently the ansatz of non-relativistic quantum chromodynamics (NRQCD) factorization was introduced in which color-octet $c \bar{c}$ states can also lead to the formation of the colorless charmonium state and thus to the charmonium production cross section.

Theoretical calculations based on the NRQCD factorization approach ${ }^{6}$ are available in leading order $^{7,8}$. In the NRQCD factorization approach the size of the coloroctet contributions, which are described by long distance matrix elements (LDME), are additional free parameters and have been determined in fits to the Tevatron data $^{9}$. The NRQCD factorization approach contains the color-singlet model which is recovered in the limit in which the long distance matrix elements for the coloroctet contributions tend to zero. For photoproduction the cross section for the color-singlet contribution was calculated to next-to-leading order in $\alpha_{s}{ }^{10}$. Charmonium production cross sections have also been calculated in the $k_{t}$ factorization approach $^{11}$. In these calculations the color-singlet model is used to describe the formation of the charmonium state.

\section{Cross Section Measurements}

Charmonium production cross sections at very high energies were first measured at the Tevatron and later in $\gamma \gamma$ collisions at LEP, and in $\gamma p$ and $e p$ collisions at HERA. Figure 5 shows data from $\mathrm{CDF}^{12}$ together with CSM calculations to leading order and fitted color-octet contributions. It can be seen that the color-octet contributions are large, leading to a good description of the data. Unfortunately those long distance matrix elements which are most important in $J / \psi$ and $\psi(2 S)$ photoproduction at HERA, are not well constrained by the Tevatron data and thus contain large uncertainties ${ }^{13}$.

In fig. $6 \mathrm{a}$ and $\mathrm{b} J / \psi$ photoproduction cross section measurements by $\mathrm{H} 1^{14}$ and ZEUS $^{1}$ are shown compared with theoretical predictions ${ }^{13}$. The $J / \psi$ data are not corrected for feed-down processes from diffractive and inelastic production of $\psi(2 S)$ mesons $(\sim 15 \%)$, the production of $b$ hadrons with subsequent decays to $J / \psi$ mesons, or feed-down from the production of $\chi_{c}$ states. The latter two contributions are estimated to contribute between $5 \%$ at medium $z$ and $30 \%$ at the lowest values of $z$. The open band in fig. 6a represents the sum of the color-singlet and 

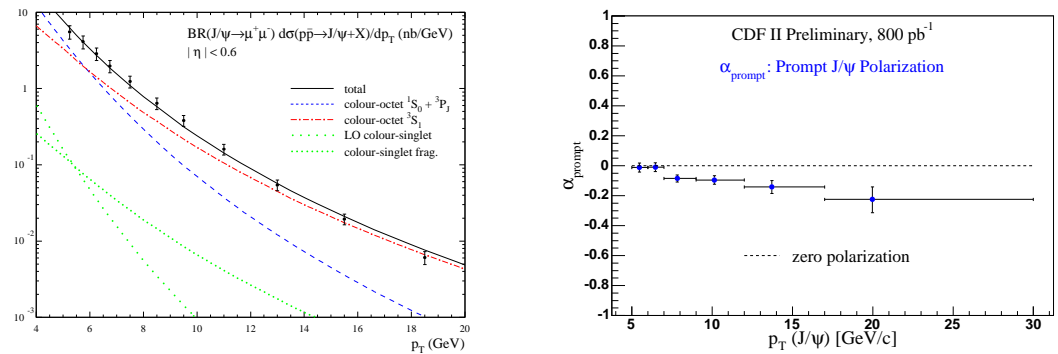

Fig. 5. Differential cross sections for the production of direct $J / \psi$ at the Tevatron as a function of $p_{t}$. The data points are CDF measurements from Run I. The dotted curves are the CSM contributions. The solid curves are the NRQCD factorization fits, and the other curves are individual color-octet contributions to the fits.

color-octet contributions, calculated in leading order NRQCD. The systematic error is dominated by the uncertainty in the long-distance color-octet NRQCD matrix elements. The shaded band shows the calculation of the color-singlet contribution to next-to-leading order in $\alpha_{s}{ }^{10}$ which describes the data quite well without the inclusion of color-octet contributions. The next-to-leading-order QCD corrections are crucial in describing the shape of the transverse-momentum distribution of the $J / \psi$.

Figures $6 \mathrm{c}$ and $\mathrm{d}$ show a comparison of the $\mathrm{H} 1$ data with the predictions from the $k_{t}$ factorization approach as implemented in the Monte Carlo generator $\mathrm{CASCADE}^{15}$. Good agreement is observed between data and predictions for $z<0.8$. At high $z$ values, the CASCADE calculation underestimates the cross section. The CASCADE predictions for the $p_{t, \psi}^{2}$ dependence of the cross section fit the data considerably better than the leading order color-singlet calculation in the collinear factorization approach (dotted curve in fig. 6b).

The analysis of electroproduction, i.e. at finite $Q^{2}$, has experimental and theoretical advantages over the photoproduction analysis. At high $Q^{2}$, theoretical uncertainties in the models are smaller than in photoproduction and resolved-photon processes are expected to be negligible. Furthermore, the background from diffractive production of charmonia is expected to decrease faster with $Q^{2}$ than the inelastic process, and the distinct signature of the scattered lepton makes the inelastic process easier to detect. The differential electroproduction cross sections for $J / \psi$ mesons from ZEUS ${ }^{16}$ and $\mathrm{H} 1^{17}$ are shown in fig. 6 e and $\mathrm{f}$ as functions of $z$. The data are compared with predictions in the framework of NRQCD at leading order in $\alpha_{s}$ taking into account diagrams of the type " $2 \rightarrow 2^{18}$, and also with predictions in the $k_{t}$ factorization approach in which only the color-singlet contribution (CS) is included ${ }^{15,19}$. The uncertainties in the NRQCD calculations are indicated in as bands. The agreement with the color-singlet model (CS), seen in fig. 6e, deteriorates when the cut $p_{t, \psi}^{* 2}>1 \mathrm{GeV}$ is applied (fig. 6f) in order to avoid regions of collinear singularities, in particular for the contributions $e+g \rightarrow e+c \bar{c}[n]+g$ with $n={ }^{1} S_{0}^{(8)}$ and ${ }^{3} P_{J}^{(8)} 18$. 

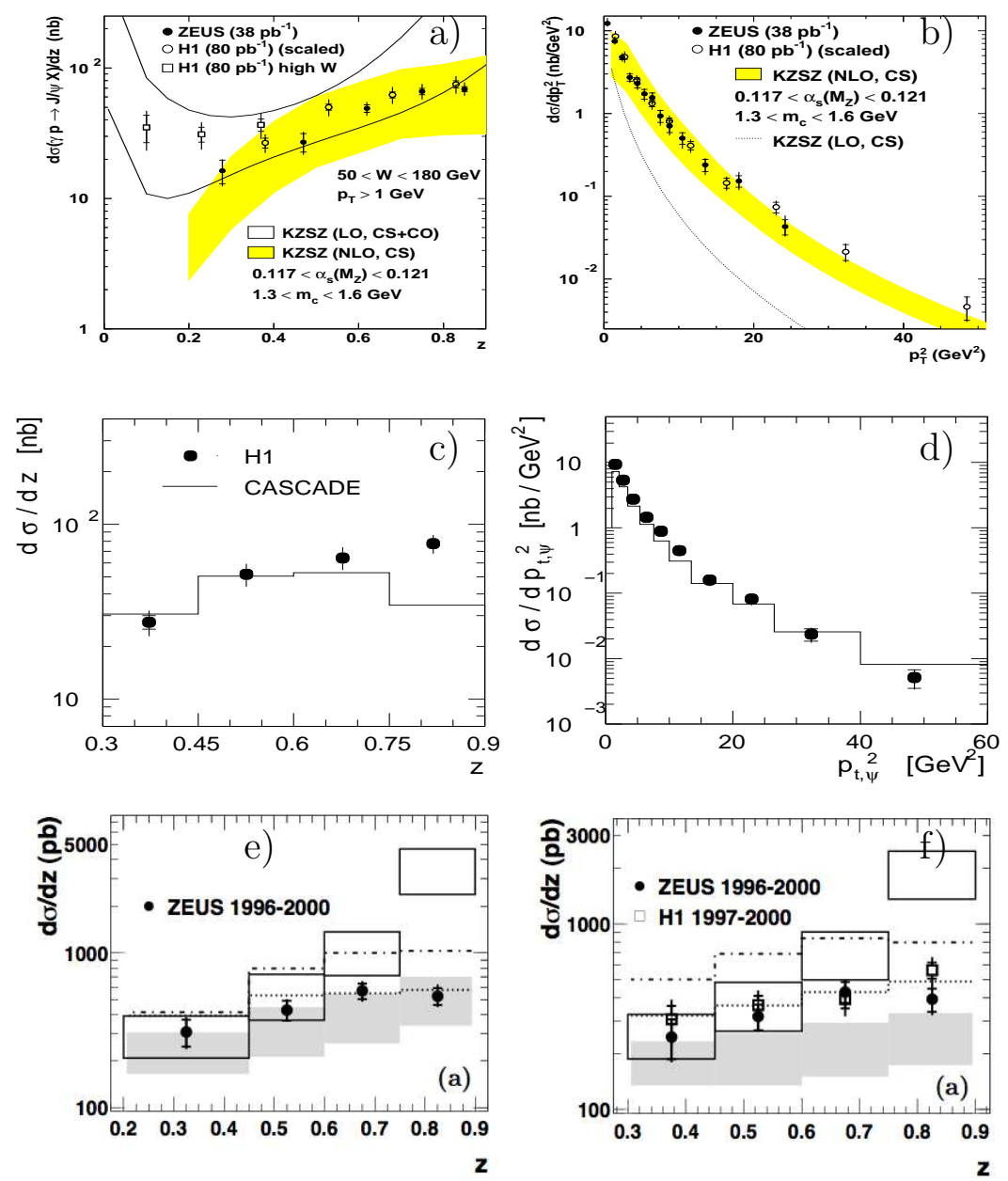

Fig. 6. a,b) The rate for inelastic $J / \psi$ photoproduction at HERA as a function of a) $z$ and b) $p_{t, \psi}$. The open band represents the LO NRQCD factorization prediction. The shaded band represents the NLO color-singlet contribution. The dotted line in b) denotes the LO color-singlet contribution. c,d) Differential Charmonium photoproduction in comparison with a prediction using the colorsinglet model and $k_{t}$ factorization as implemented in the Monte Carlo generator CASCADE. Differential electroproduction cross section $d \sigma / d z$ e) without and f) with a cut on $p_{t, \psi}^{* 2}>1 \mathrm{GeV}$. The data from ZEUS and in $\mathrm{f}$ ) also H1 are compared with the NRQCD calculation (CS+CO, open band), the color-singlet contribution (CS, shaded band), with a prediction in the $k_{t}$ factorization approach assuming the CSM (dotted line) and with the Monte Carlo generator CASCADE (dashdotted line).

NRQCD, as presently available in leading order, does not give a satisfactory description of the cross section. In contrast, the color-singlet model shows a reasonable description of the HERA data. This applies in particular to the photoproduction region where calculations to next-to-leading order perturbation theory are available. 

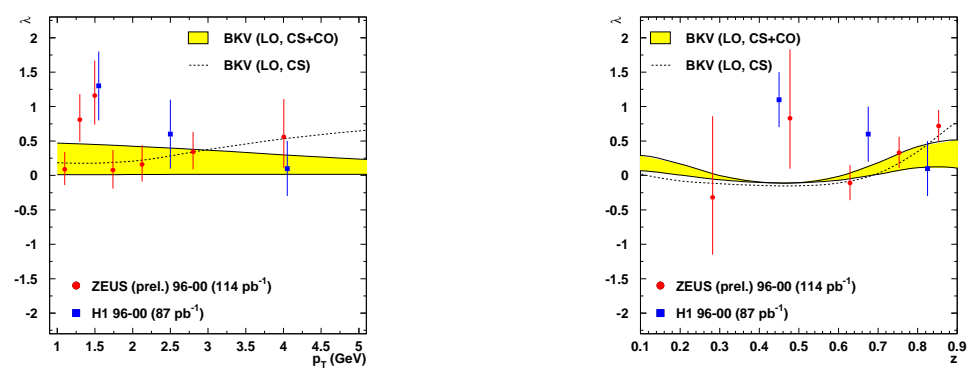

Fig. 7. Polarization parameter $\lambda$ in the target rest frame as function of $p_{t, \psi}$ and $z$. The theoretical calculations shown are from the NRQCD approach (shaded bands) with color-octet and color-singlet contributions, while the curves show the result from the color-singlet contribution separately.

\section{Polarization Measurements}

The polarization of the $J / \psi$ meson is expected to be a clear signature for the presence of color-octet contributions. Towards large transverse momenta, NRQCD predicts sizable contributions from gluon splitting, leading to an increased rate of transversely polarized $J / \psi$. The general decay angular distribution can be parameterized as $d \Gamma\left(J / \psi \rightarrow \ell^{+} \ell^{-}\right) / d \Omega \propto 1+\lambda \cos ^{2} \theta+\mu \sin 2 \theta \cos \phi+\frac{\nu}{2} \sin ^{2} \theta \cos 2 \phi$, where $\theta$ and $\phi$ refer to the polar and azimuthal angle of the three-momentum of the positive lepton with respect to a coordinate system that is defined in the $J / \psi$ rest frame $^{8}$. The parameters $\lambda, \mu, \nu$ can be calculated within NRQCD or the CSM as a function of the kinematic variables, such as $z$ and $p_{t, \psi}$.

In fig. 7 , the measurements of the parameter $\lambda$ are shown, together with the results from two LO calculations: the NRQCD prediction, including color-octet and color-singlet contributions ${ }^{8}$, and the color-singlet contribution alone. In contrast to the predictions shown in fig. 7 , in which $\lambda$ is zero or positive, the prediction of the $k_{t}$ factorization approach is that $\lambda$ should become increasingly negative toward larger values of $p_{t, J / \psi}$, reaching $\lambda \sim-0.5$ at $p_{t, \psi}=6 \mathrm{GeV}$. However, at present, the errors in the data preclude any firm conclusions. In order to distinguish between full NRQCD and the color-singlet contribution alone, measurements at larger $p_{t, \psi}$ are required. The measured values of $\nu$ (not shown), for which no prediction is available from the $k_{t}$ factorization approach, slightly favor the full NRQCD prediction.

Recently, a new measurement of the decay angular distributions of $J / \psi$ hadroproduction in $p \bar{p}$ collisions at the CDF experiment has been made available ${ }^{20}$ (fig. 5b). The result is in contradiction to a polarization increase expected from NRQCD.

\section{Outlook}

The HERA-II data (2003-2007) will comprise a factor of 4-5 more integrated luminosity, giving access to more detailed measurements with improved statistical 
precision and an increased reach in transverse momentum. In connection with improved theoretical calculations, the HERA measurements provide a fundamental test of the NRQCD factorization ansatz and the universality of the matrix elements. The HERA collider experiments H1 and ZEUS are in the process of preparing improved measurements based on the HERA-II data of $J / \psi$ and $\psi(2 S)$ production cross sections in photoproduction and in electroproduction. The $J / \psi$ decay angular distributions will be measured in photoproduction and electroproduction ${ }^{\mathrm{a}}$.

\section{Summary}

In conclusion, NRQCD, as presently available in leading order, does not give a satisfactory description of the HERA data. In contrast, the color-singlet model shows a reasonable description of the HERA data, when implemented in calculations to next-to-leading order perturbation theory or in calculations in which the $k_{t^{-}}$ factorization approach is used.

\section{References}

1. S. Chekanov et al. [ZEUS Collaboration], Eur. Phys. J. C 27 (2003) 173.

2. D. Szuba, "Vector Meson Production at HERA", Proceedings Meson 2004.

3. E. L. Berger and D. L. Jones, Phys. Rev. D 23 (1981) 1521; R. Baier and R. Rückl, Nucl. Phys. B 201 (1982) 1.

4. F. Halzen, Phys. Lett. B 69 (1977) 105; O. J. P. Eboli, E. M. Gregores and F. Halzen, Phys. Lett. B 451 (1999) 241 [hep-ph/9802421].

5. A. Edin, G. Ingelman and J. Rathsman, Phys. Rev. D 56 (1997) 7317 [hep-ph/9705311].

6. W. E. Caswell and G. P. Lepage, Phys. Lett. B 167 (1986) 437; G. T. Bodwin, E. Braaten and G. P. Lepage, Phys. Rev. D 51 (1995) 1125.

7. M. Cacciari and M. Krämer, Phys. Rev. Lett. 76 (1996) 4128 [hep-ph/9601276]; B. A. Kniehl and G. Kramer, Phys. Lett. B 413 (1997) 416 [hep-ph/9703280].

8. M. Beneke, M. Krämer, and M. Vanttinen, Phys. Rev. D 57 (1998) 4258.

9. E. Braaten, B. A. Kniehl and J. Lee, Phys. Rev. D 62 (2000) 094005.

10. M. Krämer, J. Zunft, J. Steegborn, and P. M. Zerwas, Phys. Lett. B 348 (1995) 657; M. Krämer, Nucl. Phys. B 459 (1996) 3.

11. S. P. Baranov, Phys. Lett. B 428 (1998) 377; V. A. Saleev and N. P. Zotov, Mod. Phys. Lett. A 9 (1994) 151 [Erratum-ibid. A 9 (1994) 1517].

12. F. Abe et al. [CDF Collaboration], Phys. Rev. Lett. 79 (1997) 572.

13. M. Krämer, Prog. Part. Nucl. Phys. 47 (2001) 141.

14. C. Adloff et al. [H1 Collaboration], Eur. Phys. J. C 25 (2002) 25.

15. H. Jung, Comput. Phys. Commun. 143 (2002) 100 [hep-ph/0109102].

16. S. Chekanov et al. [ZEUS Collaboration], hep-ex/0505008.

17. C. Adloff et al. [H1 Collaboration], Eur. Phys. J. C 25 (2002) 41 [hep-ex/0205065].

18. B. A. Kniehl and L. Zwirner, Nucl. Phys. B621 (2002) 337 [hep-ph/0112199].

19. A. V. Lipatov and N. P. Zotov, Eur. Phys. J. C 27 (2003) 87 [hep-ph/0210310].

20. CDF-Coll., www-cdf.fnal.gov/physics/new/bottom/060622.blessed-jpsi-spin-alignment/

21. ZEUS-Collaboration, www-zeus.desy.de/physics/phch/conf/ichep06/hfl/12/ZEUS-prel-06015.pdf

${ }^{a}$ new preliminary results were presented by the ZEUS collaboration at ICHEP06, Moscow ${ }^{21}$ 